\title{
Discovering Relevant Semantic Associations using Relationship Weights
}

\author{
S. Narayana \\ Gudlavalleru Engineering College \\ Gudlavalleru, A.P., India
}

\author{
G.P.S. Varma, Ph.D \\ Professor, S.R.K.R.Engineering \\ College, Bhimavaram
}

\author{
A. Govardhan, Ph.D \\ Professor of CSE \& Director of SIT, \\ JNTU, Hyderabad
}

\begin{abstract}
Accessing relevant pages is the primary focus of the search techniques of Web 2.0 whereas accessing relevant semantic associations is the main focus of search techniques of Web 3.0 called the Semantic Web. Discovering relevant semantic associations is especially useful in many applications such as National Security, Business Intelligence, Pharmacy, and Genetics. Semantic associations are the complex relationships between two entities such as people, places, events, publications, organizations etc. They lend meaning to information, making it understandable and actionable, and provide new and possibly unexpected insights. One of the criteria to find relevant semantic associations is based on context which captures user's domain of interest. Existing methods defines context based on the concepts or regions selected from the ontology at the schema level but not based on user interested relationships. Due to this, sometimes user gets too many associations which further require a search for relevant associations. To overcome this problem, this paper proposes a method to define the context both based on user interested concepts and the relationships so that user can get more relevant associations. To experiment the proposed method, SWETO ontology has been used and the results show that the proposed method discovers more relevant semantic associations.
\end{abstract}

\section{Keywords}

Complex Relationship, OWL, RDF, RDFS, Semantic Web, Semantic Association.

\section{INTRODUCTION}

Nowadays the Web is changing at an incredible pace, much faster than a user or even a softbot Web agent can keep up with. While new pages are being added, the content of existing pages is changing tremendously. Some pages are fairly static, others change on a regular basis, and still others change at unpredictable intervals. Due to this dynamic nature of the Web, it is extremely difficult to access relevant information from the Web. Significant research has been done on Information Retrieval Systems which lead to the development of popular search engines like Google, AltaVista, Yahoo, Bing etc. However, the main problem with the current Web infrastructure is that data on the Web is not understandable to search engine software. Just like printed paper which consist free text, a web page consists of free text as well as tags which help presentation of data to the user using a web browser. Because of this search engine software imitate human behavior in parsing and locating keywords in a web page and sometimes produce too many results for any given keyword or key phrase which further requires scan of the result set to identify relevant pages. This process is cumbersome and time consuming and sometimes leads to frustration. One of the reasons for this is the lack of "semantic information" in the content of the web page.

In many cases user might be very much interested in determining "How entity $\mathrm{X}$ is related with entity Y?" Here entities may represent people, places, events, publications, organizations and so on. For example, a politician may be related with political organization, election, legislation, state and country. He also related to other entities by different kinds of relationships like hobbies, religion, education, funding etc. Discovering the relationships between two entities gives potential information especially in applications such as National security, Business Intelligence, Genetics and Pharmacy. For example in Pharmaceutical industry it is very much useful to determine relationships among entities which typically include genes, proteins, compounds and diseases in order to discover new drugs. Current Web infrastructure is not capable to find such relationships among the entities. This is because, in current Web infrastructure web pages are connected with only one relationship called the hyper link.

To overcome the above said problems a new web infrastructure called the Semantic Web [12] is developed. Semantic Web is an extension of the current Web, enables machines to interpret and understand the data on the web. This capability of machine understandability is achieved using ontology. Ontology is a formal explicit conceptualization of a particular domain which describes the entities and how they are related with other entities. In the Semantic Web RDF [13], RDFS [14], and OWL [15] are used to specify the ontology at different expressiveness. Several communities are effectively conceptualizing domain knowledge and enabling standards for exchanging, managing and integrating data more efficiently. Due to this ongoing work, large scale repositories of semantic metadata such as WordNet (which provide a thesaurus for over 100,000 terms explained in natural language), CYC [18](which provides formal axiomating theories for many aspects of commonsense knowledge), TAP [17] (which contains information pertaining to authors, sports, companies, etc.), SWETO [16](which contains information related to cities, states and countries, air ports, companies, banks, terrorist attacks and organizations etc.) have been created and made publicly available.

Given these developments, an arena has now been set for the next generation of technologies, which will facilitate accessing of complex relationships among entities present in the semantic metadata. The complex relationships that exist between two entities are known as Semantic Associations [6] [7]. Perhaps, these relationships are at the heart of semantics [19], giving meaning to the content, and making it understandable to the machines.

When querying for semantic associations, the user is frequently overwhelmed with huge number of semantic 
associations. Hence, ranking of semantic associations is needed in order to retrieve relevant semantic associations. Several techniques have been proposed to discover relevant semantic associations involving different criteria. One criterion to retrieve relevant semantic associations is based on context. Context captures user's domain of interest based on which he/she wish to find associations. It is defined by selecting concepts or regions from the ontology at the schema level. Associations that pass through these concepts or regions are considered to be more relevant and the remaining associations are considered to be less relevant. In addition sometimes user wishes to find associations based on the relationships which are interesting to him. For example consider the domain of scientific publications where there are two relationships called author in (between researcher and publication) and cited by (between publication and author). Suppose user is interested in finding important publications, then the relationship cited by is more important than author in relationship since publications with more citations are assumed to be more important. Existing systems allows the user to define the context only with respect to the concepts or regions selected from the ontology but not with respect to the relationships which are more interesting to the user. This paper proposes a technique to define the context based on the concepts or regions selected from the ontology as well as based on the important relationships where relationship importance is determined depending on its weight assigned by the user. More weight to a relationship implies that the relationship is more interesting to the user. Using this modified context, user can get more relevant associations.

The rest of the paper is organized as follows; Section 2 reviews related work, Section 3 describes the data model and basic definitions of Semantic Associations, Section 4 explains the proposed method, the experimental results are presented in Section 5 and Section 6 draws some conclusions and possible future work.

\section{RELATED WORK}

Several methods have been proposed to discover and rank Semantic Associations. Anyanwu and Sheth et al. [6] [7] propose a method to discover and rank Semantic Associations using $\rho$-operator which checks whether or not an association is possible. If so a traversal is made in the description base. The authors use the notion of context to capture the relevant region(s) which contains potential paths. In addition a user may assign ranks to important properties in the order of importance. This allows the display of relevant associations at the top.

Shahdad Shariatmadari et al. [8] propose a technique to find semantic associations using semantic similarity measure. The authors have used $\rho$-operator to find semantic similarity using graph similarity concept. It computes the similarity between two semantic associations based on the degree of similarity of their entities and properties using subsumption function proposed by Aleman Meza et al. [1]

Anyanwu et al. [6] proposed a method called SemRank to rank semantic associations. In this method, semantic associations are ranked based on their predictability. The rank model which it uses is a rich blend of both semantic and information-theoretic techniques with heuristics that support the search process. It provides a sliding bar using which, a user can easily vary the search mode from conventional search mode to discovery search mode. The relevancy of a Semantic Association is measured based on the information content of the association which is computed based on the occurrence of edge as an event and RDF properties as outcomes. In other word, it measures property's uniqueness with respect to the other properties in the knowledge base to decide association relevancy.

Aleman Meza et al. [1] [2] propose a method to rank Semantic Associations using six types of criteria called Subsumption(components that occur at lower level in the hierarchy convey more information than those that occur at upper level), Path length(allows to select longer or shorter paths), Popularity(allows to prefer popular entities or unpopular entities based on number of incoming and outgoing edges), Rarity(allows to prefer rarely occurring or commonly occurring paths), Trust(decides the reliability of the relationship based on its origin) and Context. This method also ranks Semantic Associations using user preferences such as favour rare or common associations, popular or unpopular associations and shorter or longer associations.

Lee $M$ et al. [9] propose a method to rank Semantic Associations based on information theory and spreading activation to expand the semantic network. In this method, the results are provided that are relations between search keyword and other resources in a semantic network.

Viswanathan and Ilango et al. [5] propose a personalization approach for ranking Semantic Associations between two entities. They capture user's interest level in different domains based on their Web browsing history. The value of the user's interest level is stored in a table and based on these values the context weight of the associations is calculated and ranked.

Fabrizio Lamberti et al. [20] proposed a relation-based page rank method to rank relevant pages. In this they used relationships among the entities and the Semantic Web technologies to assign ranks to the pages in order to retrieve most relevant pages.

The main difference between the proposed method and other existing methods is that the proposed method defines the context both based on user interested concepts and relationships. Different relationships are given different weightage based on user interest and the semantic associations are ranked accordingly.

\section{BACKGROUND}

\subsection{Data Model}

On the Semantic Web, information is represented as a set of assertions called statements made up of three parts: subject, predicate, and object. The subject and the object of a statement is the resource that a statement describes, and the predicate describes a relationship between the subject and the object. The relationship is labeled with the name of the property and resource is labeled with the URI of the resource. A resource can be an entity or a literal. Object can be another resource or a literal. Assertions of this form a directed graph, with subjects and objects of each statement as nodes, and predicates as edges. This is the data model used by the Semantic Web, and it is formalized in the language called the Resource Description Framework (RDF). RDF is a World Wide Web Consortium (W3C) standard for describing Web resources. The class hierarchy of resources and property hierarchy are described in an RDF Schema (RDFS) which acts the standard vocabulary for RDF. The Web Ontology Language (OWL) extends the RDFS vocabulary with additional features. The description of relationships in OWL, adds meaning to the content and it is interpreted by search engines. 


\subsection{Semantic Associations}

The complex relationships between two entities are known as Semantic Associations [2]. The most useful Semantic Associations involve some intermediate entities and relationships. To define Semantic Associations, the formalism specified by Anyanwu et al. [7] is followed.

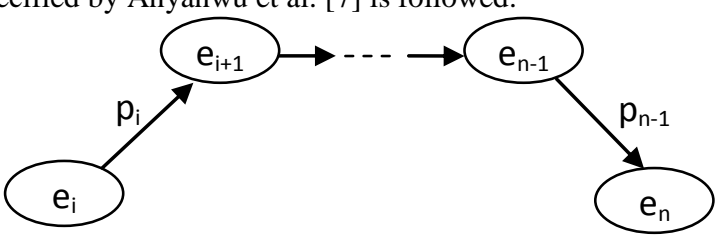

Fig 1: Semantic Association between entities $e_{i}$ and $e_{n}$

\subsubsection{Definition 1 (Semantic Connectivity)}

Two entities e1 and en are semantically connected if there exists a sequence e1, P1, e2, P2, .., en-1, Pn-1, en in an RDF graph where ei $(1 \leq i \leq n)$ are entities and $P j(1 \leq j \leq n)$ are properties. Fig. 1 shows the semantic connectivity between e1 and en

\subsubsection{Definition 2 (Semantic Similarity)}

Two entities $e_{1}$ and $f_{1}$ are semantically similar if there exist two semantic paths $\mathrm{e}_{1}, \mathrm{P}_{1}, \mathrm{e}_{2}, \mathrm{P}_{2}, \ldots, \mathrm{e}_{\mathrm{n}-1}, \mathrm{P}_{\mathrm{n}-1}, \mathrm{e}_{\mathrm{n}}$ and $\mathrm{f}_{1}, \mathrm{Q}_{1}$, $f_{2}, Q_{2}, \ldots, f_{n-1}, Q_{n-1}, f_{n}$ semantically connecting $e_{1}$ with en and $f_{1}$ with $f_{n}$ respectively, and that for every pair of properties $P_{i}$ and $\mathrm{Q}_{\mathrm{i}}, 1 \leq \mathrm{i} \leq \mathrm{n}$, either of the following conditions holds; $\mathrm{P}_{\mathrm{i}}=\mathrm{Q}_{\mathrm{i}}$ or $\mathrm{P}_{\mathrm{i}} \square \mathrm{Q}_{\mathrm{i}}$ or $\mathrm{Q}_{\mathrm{i}} \square \mathrm{P}_{\mathrm{i}}$ ( $\square$ means rdf:subPropertyOf), then two paths originating at $\mathrm{e}_{1}$ and $\mathrm{f}_{1}$, respectively, are semantically similar.

\subsubsection{Definition 3 (Semantic Association)}

Two entities $\mathrm{e}_{\mathrm{x}}$ and $\mathrm{e}_{\mathrm{y}}$ are semantically associated if $\mathrm{e}_{\mathrm{x}}$ and $\mathrm{e}_{\mathrm{y}}$ are semantically connected or semantically similar.

\section{RANKING SEMANTIC ASSOCIATIONS}

\subsection{Computing the Context based on User Interested Concepts Selected from the Ontology}

Consider the scenario where user wishes to find semantic associations between two persons in the domain of 'Politics'. Then concepts such as 'Politician', 'Political Organization', 'Government Organization' and 'Legislation' are considered to be more relevant whereas the concepts such as 'Financial Organization' and 'Terrorist Organization' are considered to be less relevant. So, user is provided facilities to define his context by selecting his interested regions from the ontology, and based on this context the associations are ranked. As an example consider the RDF graph shown in Figure 2. It shows that, the user has selected three regions belonging to 'Political Organization', 'Politician', and 'Legislation'. It also shows that there are three associations viz. the top-most association (call it as S1), the middle association (call it as S2) and the bottom-most association (call it as S3). Since all the entities of $\mathrm{S} 1$ are belonging to the user selected regions so $\mathrm{S} 1$ should be ranked high. Similarly three entities of S2 are fit in the user selected regions so $\mathrm{S} 2$ should be ranked next and none of the entities of S3 belong to user selected regions, therefore S3 should be ranked lower.

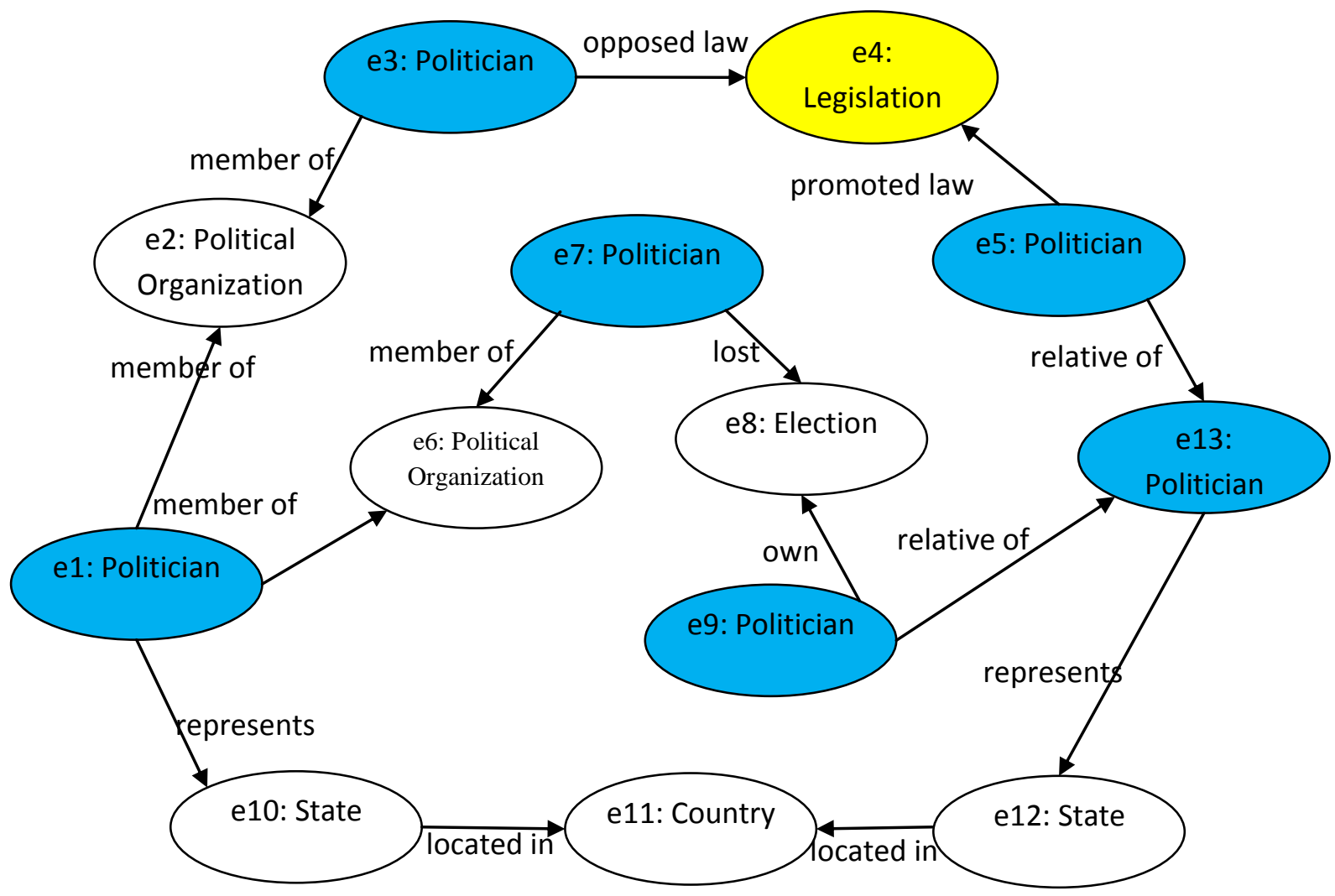

Fig 2: Part of an RDF graph showing Semantic Associations between two entities $e_{1}$ and $e_{13}$

Let $\mathrm{S}$ be a Semantic Association, c be a component of $\mathrm{S}$ which is either an entity or a relationship. Let length(S) denotes length of $\mathrm{S}$ which is the number of components present in $\mathrm{S}$ excluding first and last components. Let $\mathrm{R}_{\mathrm{i}}$ denote region $i$, which is collection of entities and relationships. Let 
$\mathrm{X}_{\mathrm{i}}$ denotes the set of components belonging to region $\mathrm{i}$ and is given as;

$$
X_{i}=\{c \mid c \in C i \wedge c \in S\}
$$

Let $Y_{i}$ denotes the set of components that does not belong to any of the regions and is given as;

$$
Y_{i}=\{c \mid c \notin C i(\forall i \mid 1 \leq i \leq n) \wedge c \in S\}
$$

Where ' $n$ ' is the number of regions selected by the user.

Now the context weight $\left(\mathrm{C}_{\mathrm{w}}\right)$ of semantic association $(\mathrm{S})$ is defined as;

$C_{w}=\frac{1}{\text { length }(S)}\left(\sum_{i=1}^{n}\left(W_{R_{i}} \times\left|X_{i}\right|\right)\right) \times\left(1-\frac{\left|Y_{i}\right|}{\text { length }(S)}\right)$

Where ' $n$ ' is the number of regions and $\mathrm{W}_{\mathrm{Ri}}$ is the weight of $\mathrm{i}^{\text {th }}$ region.

\subsection{Computing the Context based on User Interested Relationships}

Figure 2 shows part of an RDF graph from SWETO [10] ontology.

In this there are three associations as given below;

1. e1:Politician $\rightarrow$ member of $\rightarrow$ e2:PoliticalOrganization $\leftarrow$ member of $\leftarrow$ e3:Politician $\rightarrow$ opposed law $\rightarrow$ e4:Legislation $\leftarrow$ promoted law $\leftarrow$ e5:Politician $\rightarrow$ relative of $\rightarrow$ e13:Politician

2. e1:Politician $\rightarrow$ member of $\rightarrow$ e6: PoliticalOrganization $\leftarrow$ member of $\leftarrow$ e7:Politician $\rightarrow$ lost $\rightarrow$ e8:Election $\leftarrow$ own $\leftarrow$ e9:Politician $\rightarrow$ relative of e13:Politician

3. e1:Politician $\rightarrow$ represents $\rightarrow$ e10:State $\rightarrow$ located in $\rightarrow$ e11:Country $\leftarrow$ located in $\leftarrow$ e12:State $\leftarrow$ represents $\leftarrow$ e13:Politician

Suppose, a user is interested to find associations between two entities e 1 and e 13 with respect to promotion and opposition of laws, then association 1 would be more relevant to him where as association 3 would be less relevant. Similarly if he wishes to find associations with respect to winning and losing of elections, then association 2 would be more relevant and the other two relations are considered to be less relevant. Here relevance of associations is mainly depending on the relationships which connect the entities. In this paper, a new criterion called "relationship weight $\left(\operatorname{Rel}_{\mathrm{p}}\right)$ " is used to capture importance of relationships. The relationship weight $\left(\operatorname{Rel}_{\mathrm{w}}\right)$ of a semantic association (S) is computed as follows;

$$
\operatorname{Rel}_{w}(S)=\frac{1}{m} \sum_{i=1}^{m} w_{i}
$$

Where ' $\mathrm{m}$ ' is the number of relationships in $\mathrm{S}$ and $\mathrm{w}_{\mathrm{i}}$ is weight of $\mathrm{i}^{\text {th }}$ relationship. The system allows the user to give weights to relationships based on his interest. Since the number of relationships to be weighted are very less (hardly 15), it is easy to give weights to relationships. More weight to a relationship implies that the relationship is more interesting to the user.
4.3 Computing the Overall Context Weight The overall context weight of a semantic association ( $\mathrm{S}$ ) is now computed from (3) and (4) as;

$$
C_{S}=C_{w}+\operatorname{Rel}_{w}
$$

Since the overall context weight is computed in terms of the context defined based on the concepts selected from the ontology at the schema level and based on the user interested relationships it will capture user's domain of interest more effectively thus produce more relevant semantic associations.

Other criteria proposed by Aleman Meza et al. [3] are used along with the context to rank semantic associations in order to get more relevant associations. These criteria are described below.

\subsubsection{Subsumption Weight Ss}

In an RDF graph, entities that occur at lower level in the hierarchy are treated as more specialized entities than the entities that occur at higher levels. Thus, lower level entities convey more meaning. So Associations that consists these entities are more relevant.

\subsubsection{Path Weight Ls}

Sometimes, user might be interested in finding shorter associations, yet in other cases he may wish to find longer associations. So user can determine which association length influences most.

\subsubsection{Popularity Weight Ps}

Entity popularity is defined based on the number of incoming and outgoing edges the entity has. Associations that contain popular entities are considered popular associations. Hence, user has to select whether 'favour more popular associations or favour less popular associations' based on his interest.

\subsubsection{Rarity Weight Rs}

In some situations, user might be interested in either rarely occurring events or commonly occurring events. For example, in money laundering user may be interested in commonly occurring events because money launderers' perform several common transactions to escape from law. So user is allowed to select 'favour rare or common associations' according to his interest.

\subsubsection{Trust Weight Ts}

The entities and relationships in a Semantic Association come from different sources. Some sources may be more trusted and some sources may be less trusted. So trust value is assigned to components in an association based on the source from which it is coming.

The overall ranking formula for ranking Semantic Association, SA, is given as;

$$
\begin{gathered}
R_{S A}=k_{1} \times C_{S}+k_{2} \times S_{S}+k_{3} \times L_{S}+k_{4} \times P_{S}+k_{5} \times R_{S} \\
+k_{6} \times T_{S}
\end{gathered}
$$

In this, $\mathrm{k} 1+\mathrm{k} 2+\mathrm{k} 3+\mathrm{k} 4+\mathrm{k} 5+\mathrm{k} 6 \leq 1$ and is required to fine-tune the ranking of Semantic Associations.

\section{EXPERIMENTAL RESULTS}

To demonstrate the effectiveness of proposed system, SWETO (Semantic Web Technology Evaluation ontology) test-bed has been used. SWETO is a well-known and populated ontology consisting of real world entities related to cities, states and countries, air ports, companies, banks, terrorist attacks and organizations, persons and researchers, scientific publications, journals, conferences and books. In 
addition to ranking criteria various other criteria such as 'Favor short or long associations', 'Favor popular or unpopular entities', 'Favor rare or common associations' and Context were used to rank semantic associations. Semantic associations are ranked by the system as well as manually.

\subsection{User Interface}

User Interface is a web based application using Servlet and Apache Tomcat. Using this interface, user can specify two entities between which he/she wish to find semantic associations. Prior to the ranking, user can select his interested contextual regions from the ontology using a touch graph like interface and assign weights to relationships based on his interest using another interface to define the context. Additionally, user can customize his ranking criteria by assigning weights to each individual criterion. The system then finds and ranks semantic associations using the criteria as described above. Ranked Semantic Association results are shown in Table 1.

Table 1: Top 15 Ranked Semantic Associations

\begin{tabular}{|c|c|c|}
\hline Rank & Association & $\begin{array}{l}\text { Ranking } \\
\text { Score }\end{array}$ \\
\hline 1 & $\begin{array}{l}\text { George W. Bush-promoted law-PATRIOT Act-opposed law-Edward Kennedy-promoted law-No } \\
\text { Child Left Behind Act-passed law-U.S. Senate-member of-Craig Thomas }\end{array}$ & 1.006 \\
\hline 2 & $\begin{array}{l}\text { George W. Bush-signed by-No Child Left Behind Act-promoted law-Edward Kennedy-opposed law- } \\
\text { PATRIOT Act-passed law-U.S. Senate-member of-Craig Thomas }\end{array}$ & 1.002 \\
\hline 3 & $\begin{array}{l}\text { George W. Bush-signed by-PATRIOT Act-opposed law-Edward Kennedy-promoted law-No Child } \\
\text { Left Behind Act-passed law-U.S. Senate-member of-Craig Thomas }\end{array}$ & 1.002 \\
\hline 4 & George W. Bush-promoted law-PATRIOT Act-passed law-U.S. Senate-member of-Craig Thomas & 0.986 \\
\hline 5 & $\begin{array}{l}\text { George W. Bush-promoted law-PATRIOT Act-opposed law-Edward Kennedy-member of-U.S. } \\
\text { Senate-member of-Craig Thomas }\end{array}$ & 0.968 \\
\hline 6 & $\begin{array}{l}\text { George W. Bush-signed by-PATRIOT Act-opposed law-Edward Kennedy-member of-U.S. Senate- } \\
\text { member of-Craig Thomas }\end{array}$ & 0.967 \\
\hline 7 & $\begin{array}{l}\text { George W. Bush-signed by-No Child Left Behind Act-promoted law-Edward Kennedy-member of- } \\
\text { U.S. Senate-member of-Craig Thomas }\end{array}$ & 0.952 \\
\hline 8 & George W. Bush-signed by-PATRIOT Act-passed law-U.S. Senate-member of-Craig Thomas & 0.95 \\
\hline 9 & $\begin{array}{l}\text { George W. Bush-signed by-No Child Left Behind Act-passed law-U.S. Senate-member of-Craig } \\
\text { Thomas }\end{array}$ & 0.932 \\
\hline 10 & $\begin{array}{l}\text { George W. Bush-promoted law-PATRIOT Act-passed law-U.S. Senate-member of-Elizabeth Dole- } \\
\text { member of-Republican Party-member of-Craig Thomas }\end{array}$ & 0.684 \\
\hline 11 & $\begin{array}{l}\text { George W. Bush-promoted law-PATRIOT Act-passed law-U.S. Senate-member of-John McCain- } \\
\text { member of-Republican Party-member of-Craig Thomas }\end{array}$ & 0.68 \\
\hline 12 & $\begin{array}{l}\text { George W. Bush-promoted law-PATRIOT Act-passed law-U.S. Senate-member of-Jon Kyl-member } \\
\text { of-Republican Party-member of-Craig Thomas }\end{array}$ & 0.672 \\
\hline 13 & $\begin{array}{l}\text { George W. Bush-promoted law-PATRIOT Act-passed law-U.S. Senate-member of-Chuck Grassley- } \\
\text { member of-Republican Party-member of-Craig Thomas }\end{array}$ & 0.672 \\
\hline 14 & $\begin{array}{l}\text { George W. Bush-promoted law-PATRIOT Act-passed law-U.S. Senate-member of-Mel Martinez- } \\
\text { member of-Republican Party-member of-Craig Thomas }\end{array}$ & 0.672 \\
\hline 15 & $\begin{array}{l}\text { George W. Bush-promoted law-PATRIOT Act-passed law-U.S. Senate-member of-Lamar Alexander- } \\
\text { member of-Republican Party-member of-Craig Thomas }\end{array}$ & 0.672 \\
\hline
\end{tabular}




\subsection{Preliminary Results}

To demonstrate the effectiveness of the proposed method, we have compared the system ranking with the user-human ranking between two entities George W. Bush and Craig Thomas which is shown in figure 3 . The $\mathrm{x}$-axis shows ranking of semantic associations by the proposed method and y-axis shows user-human ranking which is assigned by the users manually. A group of five graduate students belonging to Computer Science Department who are not familiar with this research were selected and they have given the semantic association results. Together with these results, they have given the ranking criteria (i.e. context, Favor short or long associations, Favor popular or unpopular entities, and Favor rare or common associations). They have also given the types of entities and relationships in the semantic associations to appropriately judge whether an association is relevant to the provided context. Based on these ranking criteria, the five graduate students rank the given associations and this is compared with the system ranking using Spearman's foot rule distance measure to measure the similarity between the proposed system ranking and the user-human ranking.

Spearman's Foot rule distance measure is given as.

$$
D(\text { system, human })=\sum_{i=1}^{n}\left|R_{\text {isystem }}-R_{\text {ihuman }}\right|
$$

Spearman's Foot rule Coefficient

$$
C=1-\frac{4 D}{n^{2}}
$$

Based on the results, the average correlation coefficient between the proposed system ranking and the user-human ranking is 0.72 . Since the average correlation coefficient between the proposed system ranking and the user-human ranking is greater than 0.50 , the proposed system ranking and the user-human ranking are highly correlated. In information retrieval context, precision represents the fraction of retrieved associations that are relevant to the search. Figure 4 shows the comparison of precision rate between the proposed system and other existing methods. It shows that precision rate of the proposed method is higher than the existing methods.

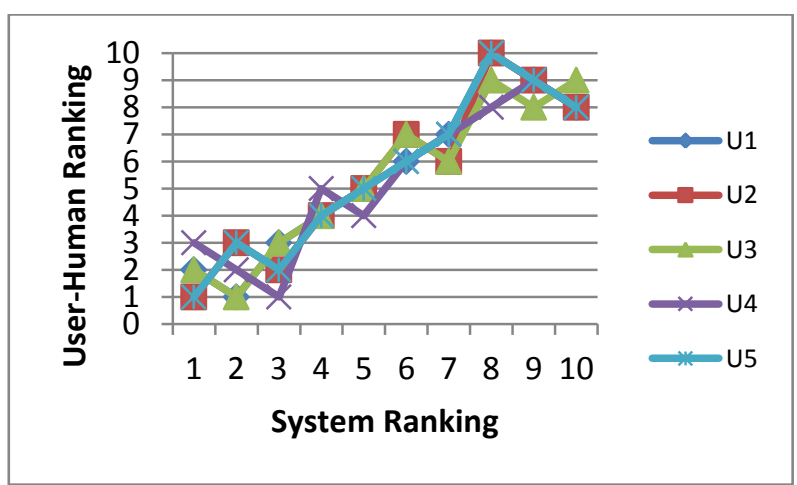

Fig 3: Comparison of proposed system ranking with userhuman ranking

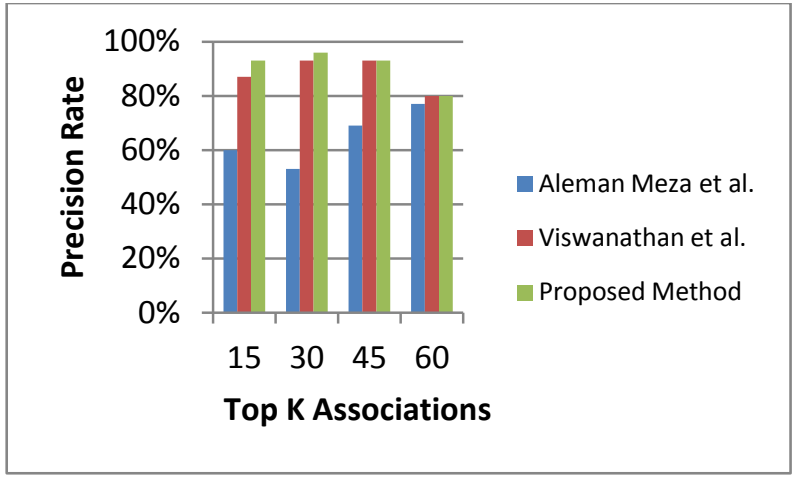

Fig 4: Comparison of precision rate of proposed method with existing methods

\section{CONCLUSION}

Finding Semantic Associations between two entities is very useful, especially in applications such as National Security, Business Intelligence, Genetics and Pharmaceutical research. Given two entities, there exist a huge number of associations. Moreover these associations pass through different intermediate entities involving different kinds of relationships. Hence, new techniques are required to find relevant Semantic Associations. This paper proposes a new criterion called Relationship Weight as one of the parameters to discover relevant Semantic Associations. The ranking of Semantic Associations of the proposed method with existing methods is compared using Spearman's Foot rule. The average correlation coefficient of proposed method is 0.72 which is greater than other existing methods. Since the average correlation coefficient is greater than 0.5 , the proposed system ranking is highly correlated with user-human ranking. When size of RDF graph grows, it is difficult for the user to specify context and assign weights to relationships through the user interface. Hence, in the coming years, a methodology is to be developed to make the system learn the above said features and assign ranks to Semantic Associations.

\section{REFERENCES}

[1] Aleman-Meza Bonerges, Halaschek-Wiener Christian, Arpinar IB, Ramakrishnan Cartic, Sheth Amit (2005). Ranking Complex Relationships on the Semantic Web. IEEE Internet Computing 9(3); 37-44. Doi:10.1109/MIC.200.63.

[2] Aleman-Meza, B., Halaschek, C., Arpinar, I. B., and Sheth, A. "Context-Aware Semantic Association Ranking." First International Workshop on Semantic Web and Databases, Berlin, Germany, 33-50

[3] Sheth, A. P., Aleman-Meza, B., Arpinar, I. B., Halaschek, C., Ramakrishnan, C., Bertram, C., Warke, Y., Avant, D., Arpinar, F. S., Anyanwu, K., and Kochut, K. (2005a). "Semantic Association Identification and Knowledge Discovery for National Security Applications." Journal of Database Management, 16(1), 33-53.

[4] Halaschek, C., Aleman-Meza, B., Arpinar, I. B., and Sheth, A. P. "Discovering and Ranking Semantic Associations over a Large RDF Metabase." 30th International Conference on Very Large Data Bases, Toronto, Canada.

[5] V Viswanathan, $\mathrm{K}$ Ilango: Ranking semantic relationships between two entities using personalization in contest specification. Informain Sciences, Elsevier, 
207 (2012) 35-49.

[6] Anyanwu Kemafor, Angela Maduko, Sheth Amit. SemRank: ranking complex relationship search results on the Semantic Web, in: Proc. of the 14th International World Wide Web Conference, ACM Press, 2005, pp. 117-127.

[7] Anyanwu Kemafor, Sheth Amit. $\rho$-operator: Discovering and Ranking Semantic Associations on the Semantic Web, ACM SIGMOD Record, v. 31 n.4, December 2002.

[8] Shahdad Shariatmadari, Ali Mamat, Ibrahim Hamidah, Mustapha Norwati (2008). SwSim:Discovering semantic similarity association in semantic web. Proceedings of International Symposium on ITSim 2008, 1-4.

[9] Myungjin Lee, Wooju Kim. Semantic association search and rank method based on spreading activation for the Semantic Web, in: IEEE International Conference on Industrial Engineering and Engineering Management, 2009, pp. 1523.

[10] Myungjin Lee, Wooju Kim, Sangun Park. Searching and ranking method of relevant resources by user intention on the Semantic Web, Expert Systems with Applications 39 (2012) 4111-4121.

[11] M.-E. Vidal, L. Rashid, L. Ibabez, J. Rivera, H. Rodrogiez, E. Ruckhaus, A ranking-based approach to discover semantic association between linked data, in: The 2nd International Workshop on Inductive Reasoning and Machine learning for the Semantic Web, 2010, pp.18-29.

[12] Berners-Lee, T., Hendler, J., and Lassila, O. (2001). "The Semantic Web - A new form of Web content that is meaningful to computers will unleash a revolution of new possibilities." Scientific American, 284(5), 34.

[13] Lassila Ora and Swick R. Resource Description Framework (RDF) Model and Syntax Specification, W3C Recommendation. 1999.

[14] Brickley D and Guha RV. Resource Description Framework (RDF) Schema Specification 1.0, W3C Candidate Recommendation. 2000.

[15] Web Ontology Language, http://www.w3.org/2004/OWL/, 2004.

[16] Aleman-Meza, B., Halaschek, C., Sheth, A., Arpinar, I. B., and Sannapareddy, G. "SWETO: Large-Scale Semantic Web Test-bed." 16th International Conference on Software Engineering and Knowledge Engineering (SEKE2004): Workshop on O1ntology in Action, Banff, Canada, 490-493.

[17] Guha, R. V., and McCool, R. (2003). "TAP: A Semantic Web Test-bed." Journal of Web Semantics, 1(1), 81-87.

[18] Opencyc. http://sw.opencyc.org

[19] Sheth, A. P., Arpinar, I. B., and Kashyap, V. (2003). "Relationships at the Heart of Semantic Web: Modelling, Discovering and Exploiting Complex Semantic Relationships." Enhancing the Power of the Internet Studies in Fuzziness and Soft Computing, M. Nikravesh, B. Azvin, R. Yager, and L. A. Zadeh, eds., SpringerVerlag. 2003.

[20] Fabrizio Lamberti, Member, IEEE, Andrea Sanna, and Claudio Demartini. A Relation-Based Page Rank Algorithm for Semantic Web Search Engines, IEEE TRANSACTIONS ON KNOWLEDGE AND DATA ENGINEERING, VOL. 21, NO. 1, JANUARY 2009, pp. 123-136. 